\title{
Sentinel Lymph Node Biopsy in Penile Cancer: A Comparative Study Using Modified Inguinal Dissection
}

\author{
Ubirajara Ferreira, Marco A.V. Ribeiro, Leonardo O. Reis, Alessandro Prudente, Wagner E. \\ Matheus
}

Division of Urology, School of Medicine, University of Campinas, Campinas, Sao Paulo, Brazil

\begin{abstract}
Introduction: In the case of clinically negative inguinal regions in penile cancer, the treatments proposed might vary from careful observation to radical dissection for all patients. We evaluated the effectiveness of the sentinel lymph node biopsy using lymphoscintigraphy in patients with penile cancer and at least one negative inguinal region.

Materials and Methods: In 18 patients, biopsy of the sentinel lymph node from the 32 negative inguinal regions and modified radical lymphadenectomy in these regions regardless of the biopsy results was performed. Clinical staging, pathological results of the sentinel and the other lymph nodes removed during lymphadenectomy, tumor behavior, local and inguinal recurrence and specific disease mortality were accessed.

Results: The mean age of the study sample was 57.7 years ( 44 - 81 years) and the sentinel lymph node presented $0 \%$ false negative $66 \%$ sensitivity, and $79.3 \%$ specificity when compared with the modified inguinal lymphadenectomy as the gold standard treatment.

Conclusion: Sentinel lymph node biopsy is a feasible method of assessing the presence of regional metastasis in patients with penile cancer and clinically negative inguinal regions. However, the optimal lymphoscintigraphy technique is still in evolution and requires further optimization at high volume centers.
\end{abstract}

Key words: penile cancer; sentinel lymph node; inguinal; lymphoscintigraphy

Int Braz J Urol. 2008; 34: 725-33

\section{INTRODUCTION}

While penile cancer is a rare disease in developed countries, its rate in underdeveloped countries is in fact the contrary (1). Disease treatment becomes a challenge when procedures related to prophylactic dissection of regional lymph nodes are evaluated.

When lymphatic metastasis in the inguinal region is clinically evident, classical radical lymph node dissection is the recommended treatment for improved overall survival and quality of life (1).

Lopes et al. comparing clinical and pathological features in penile cancer patients, found that the sensitivity, specificity, positive and negative predictive values, and effectiveness of clinical procedures for assessment of metastases were 66.7, 52.3, 60.8, 58.6 and $59.9 \%$, respectively. On multivariate analysis of pathological factors only lymphatic $(\mathrm{p}=0.0008)$ and venous $(\mathrm{p}=0.0410)$ penile embolizations were significantly associated with risk of lymph node metastases (2).

In the case of clinically negative inguinal regions, the treatments proposed might vary from careful observation to radical dissection for all patients with intermediary solutions such as sentinel lymph node biopsy and modified dissection of the inguinal region with preservation of some structures and lymph nodes (3).

The principle of identifying the first drainage lymph node in the affected area and based on its 
pathological assessment defining the need for more aggressive interventions seems to be an important and interesting procedure. This may be the reason why it has been the subject of several articles that demonstrate experience in this procedure or aim at assessing the validity of this test and its morbidity in relation to other interventions $(4,5)$.

The purpose of our study is to evaluate the effectiveness of the sentinel lymph node biopsy using lymphoscintigraphy in patients with penile cancer and at least one negative inguinal region.

\section{MATERIALS AND METHODS}

A prospective study on 18 patients who had penile cancer and at least one clinically negative inguinal region was conducted between May 2000 and July 2005 . This study was approved by Institutional Review Board.

All the patients, after signature of informed consent, had undergone partial penectomy, biopsy of the sentinel lymph node from the negative inguinal regions and modified radical lymphadenectomy as proposed by Catalona (1988) in these regions regardless of the biopsy results (3).

Biopsy of the sentinel lymph node and classical radical lymphadenectomy were performed in the positive inguinal regions.

Patients were classified according to surgical staging and histological level based on the TNM 2002 system proposed by the UICC/AJCC (6).

Patients underwent lymphoscintigraphy with ${ }^{99 m}$ technetium-labelled nanocolloid, which was injected intradermally around the tumor or into the distal penile shaft skin.

Four hours later, the sentinel lymph node was identified during surgery using a hand-held $\gamma$-probe. Complementary methods such as the peri lesion methylene blue injection were not used in this protocol.

Data assessment included clinical staging, pathological results of the sentinel lymph node and the other lymph nodes removed during modified lymphadenectomy as well as post-surgical tumor behavior, verifying local and inguinal recurrence and specific disease mortality.

\section{RESULTS}

The mean age of the study sample was 57.7 years (44 - 81 years) and the initial clinical assessment revealed that four patients had a positive inguinal region, which left 32 inguinal regions to be studied using the proposed method as clinically affected regions were excluded from the study.

In 6 of the 32 inguinal regions studied, the lymphoscintigraphy did not detect the sentinel lymph node when the gamma camera and the intraoperative portable probe were used, which meant that 26 inguinal regions with sentinel lymph nodes were identified and a total of 52 lymph nodes were removed at this stage. It should be underscored that one of the six undetected lymph node inguinal regions was in a patient with a clinically contralateral positive inguinal region.

The results of the pathological study of the sentinel lymph nodes revealed that only two inguinal regions of the 26 regions studied were affected $(7.7 \%)$.

It should be emphasized that of these two clinically negative regions with positive sentinel lymph nodes, one of them was in a patient with a clinically positive contralateral inguinal region.

When the pathological study results of the modified lymph node dissection specimens in 32 clinically negative inguinal regions were assessed, three were found to be positive. The positive result indicated the need for radical procedure.

Two of them were from inguinal regions with positive sentinel lymph nodes and one was found among the six clinically negative inguinal regions without sentinel lymph node detection. Furthermore, one of the two positive sentinel nodes was the only positive nodes in this dissection. They were 0.8 and $0.6 \mathrm{~cm}$ large and the total of node dissected in theses regions were 7 and 10, respectively.

In the 24 inguinal regions with negative sentinel lymph node, no diagnostic changes occurred in any of them during lymph node dissection. Therefore, when we compared this method with the modified inguinal lymphadenectomy as the gold standard treatment, we found these values: $0 \%$ false negative, $66 \%$ sensitivity and $79.3 \%$ specificity. It is important to highlight that this false negative rate does not consider 
the patient without detection of sentinel lymph node and positive lymphatic dissection.

Some more relevant data can be cited: a total of 297 lymph nodes were dissected, 157 on the right and 140 on the left, a mean number of 8.25 lymph nodes per region studied.

A study of the dissected lymph nodes confirmed the diagnosis of regional metastasis in all four clinically positive regions. Moreover, in all the patients who underwent classical radical dissection due to lymph node positivity, the positivity of the lymph nodes removed did not surpass the limits proposed by the modified dissection technique.

In four clinical positive regions, we found 10 positive nodes out of 35 dissected. These were mean $2.2 \mathrm{~cm}$ large and none was adhered to the adjacent tissues.

An evaluation of the distribution of these patients according to staging and histological grade revealed that six patients were pT1G1, 8 were pT2G1, 3 were pT2G2 and 1 was pT3G3. Metastasis was not detected in patients with $\mathrm{T} 1$ primary lesions.

The follow-up period ranged from 8 to 58 months, with a mean follow-up period of 28.3 months.

Two patients presented regional disease recurrence that was confirmed by the pathological exam and both patients presented positive surgical staging for metastasis in the inguinal regions. Both patients died two and four months after recurrence. These patients had presented with positive groins at initial diagnosis and were submitted to radical lymphadenectomy.

Another 81-year-old patient died three months after surgery but the cause was not directly related to the disease.

Table-1 demonstrates the above-described results.

\section{COMMENTS}

The literature demonstrates that after resection, $20 \%$ of clinically negative inguinal regions in patients with penile cancer have proved to be positive (7). This data underscores the importance of proposing some kind of treatment for patients in this condition, especially since prognosis is poor in patients with delayed diagnosis of lymph node metastasis (8).

Neglected regional adenopathy is not uncommon in underdeveloped countries after penile cancer treatment and in advanced cases, the inguinal mass can reach large sizes and little can be offered as a curative treatment (9).

Considering that groin is the first site of lymphatic dissemination of penile squamous cell carcinoma, inguinal involvement is one of the most important factors in survival prognosis and it is very common to lost follow-up of these patients in underdeveloped countries, we offer lymphadenectomy concomitant to penectomy in our institution (9).

On the other hand, lymph node dissection in the inguinal region can cause up to $80 \%$ morbidity $(10,11)$. A better assessment can be obtained with a biopsy of a single or small group of sentinel lymph nodes as it allows for finer sections of the specimen and an immunohistochemical study whenever required (5).

In view of the fact that in $50 \%$ of positive cases, the sentinel lymph node was the only lymph node affected after ample dissection, it has been suggested that on the basis of parameters such as size of metastasis and histological grade of cell differentiation, only the sentinel lymph node should be removed even when it is positive $(8,12)$.

Our study demonstrated that in two cases of sentinel lymph node positivity, the disease was restricted only to the sentinel lymph node in one case.

Another significant data was the false negative percentage, which in our study was $0 \%$ but in the literature is approximately 11 to $18 \%(4,5,13)$. We could have obtained similar results if the study sample was larger.

However, there were no cases of sentinel lymph node detection by the gamma camera without confirmation by the intraoperative portable probe, but in conformity occurred in $30 \%$ of the cases in the literature (4). This may be due to a technical change brought about by the lymphoscintigraphy, which was performed on the same morning as the surgery, indicating that the probe was used a few hours after the initial exam. Moreover, this technical change eliminated the need for a complementary exam with methylene blue $(14,15)$. 
Table 1 - Cases studied and results obtained.

\begin{tabular}{lccccccccc}
\hline Patient & Stage & Grade & Age & Right & Left & Right & Left & Node & Follow-up \\
\hline 1 & PT2 & II & 60 & excluded & no captation & excluded & $(-)$ & N1 & 58 \\
2 & PT1 & I & 55 & $(-)$ & $(-)$ & $(-)$ & $(-)$ & N0 & 55 \\
3 & PT2 & I & 50 & $(-)$ & $(-)$ & $(-)$ & $(-)$ & N0 & 51 \\
4 & PT1 & I & 51 & no captation & $(-)$ & $(-)$ & $(-)$ & N0 & 47 \\
5 & PT1 & I & 63 & $(-)$ & $(-)$ & $(-)$ & $(-)$ & N0 & 43 \\
6 & PT1 & I & 56 & $(-)$ & $(-)$ & $(-)$ & $(-)$ & N0 & 33 \\
7 & PT2 & I & 44 & $(-)$ & $(-)$ & $(-)$ & $(-)$ & N0 & 30 \\
8 & PT2 & II & 49 & $(-)$ & $(-)$ & $(-)$ & $(-)$ & N0 & 29 \\
9 & PT2 & I & 81 & $(-)$ & excluded & $(-)$ & excluded & N1 & 26 \\
10 & PT1 & I & 50 & $(-)$ & $(-)$ & $(-)$ & $(-)$ & N0 & 24 \\
11 & PT2 & I & 45 & $(+)$ & no captation & $(+)$ & $(+)$ & N2 & 21 \\
12 & PT2 & I & 70 & $(-)$ & no captation & $(-)$ & $(-)$ & N0 & 19 \\
13 & PT2 & I & 52 & $(-)$ & $(-)$ & $(-)$ & $(-)$ & N0 & 18 \\
14 & PT2 & I & 63 & $(-)$ & no captation & $(-)$ & $(-)$ & N0 & 15 \\
15 & PT2 & II & 65 & $(-)$ & excluded & $(-)$ & excluded & N1 & 12 \\
16 & PT2 & I & 72 & $(-)$ & $(-)$ & $(-)$ & $(-)$ & N0 & 10 \\
17 & PT3 & II & 66 & excluded & $(+)$ & excluded & $(+)$ & N2 & 10 \\
18 & PT1 & I & 47 & no captation & $(-)$ & $(-)$ & $(-)$ & N0 & 8 \\
& & & 57.7 & & & & & & 28.3 (SD 16.2) \\
\hline
\end{tabular}

$S D=$ standard deviation .

Other noninvasive procedures have been used to detect inguinal metastases for penile cancer, including the use of lymphotropic nanoparticles enhanced magnetic resonance imaging (16).

Recently Horenblas et al. studied 50 patients with penile cancer and negative groins. These patients were submitted to SPEC-CT and lymphoscintigraphy utilizing the Daseler's five zones, that divide the groin region in four quadrants and one central zone on sapheno-femoral junction, they found that all sentinel nodes were in superior or central zones. In conclusion, they suggested that the dissection should be limited on and above the sapheno-femoral junction (17).

Some authors of Latin America have proposed endoscopic inguinal lymphadenectomy with decreased surgical morbidity, such as skin necrosis, wound infections and lymphedema. Although new endoscopic approaches to perform inguinal lymphadenectomy may decrease postoperative morbidity without compromising oncological control, sentinel lymph node biopsy may help improve patient selection for inguinal lymphadenectomy, preventing unnecessary procedures or indicating earlier surgery when lymph nodes are not yet palpable $(18,19)$.

Although these novel noninvasive tests appear sensitive in preliminary reports, their ultimate value awaits further validation.

One of our cases of lymphatic metastasis with a clinically negative region occurred in a patient with an undetected sentinel lymph node. This resulted in a $66 \%$ test sensitivity that was comparable with the literature and reinforced the need for surgical exploration of the inguinal region without lymphoscintigraphic detection of the sentinel lymph node $(4,5,20,21)$.

Although the sentinel biopsy decreases the morbidity in penile cancer treatment, there are limitations to a less aggressive approach and patients with undetected sentinel lymph node being considered a method limitation (22). 
On the other hand, false-negative rate is encouraging and suggests that with experience and technical evolution lymphoscintigraphy could become a standard procedure requiring further optimization at high volume centers.

Taking into consideration that Catalona procedure fails to identify until $15 \%$ of patients developing late metastasis, new methods are warranted in the future (3).

Considering the learning curve associated with lymphoscintigraphy and that penile cancer is a rare disease, the optimal lymphoscintigraphy technique is still in evolution, but it may be a very good option in the future.

\section{CONCLUSION}

Sentinel lymph node biopsy is a feasible method of assessing the presence of regional metastasis in patients with penile cancer and clinically negative inguinal regions. It does not appear to decrease oncological outcomes.

However, the optimal lymphoscintigraphy technique is still in evolution and requires further optimization at high volume centers.

\section{CONFLICT OF INTEREST}

None declared.

\section{REFERENCES}

1. Wroclawski ER, Sampaio FJ: Guia prático de Uropatologia. Relatório da Reunião de Consenso. Int Braz J Urol. 2003; 29 (suppl 1): 44 -50.

2. Lopes A, Hidalgo GS, Kowalski LP, Torloni H, Rossi BM, Fonseca FP: Prognostic factors in carcinoma of the penis: multivariate analysis of 145 patients treated with amputation and lymphadenectomy. J Urol. 1996; 156: $1637-42$.

3. Catalona WJ: Modified inguinal lymphadenectomy for carcinoma of the penis with preservation of saphenous veins: technique and preliminary results. J Urol. 1988; 140: 306-10.

4. Perdonà S, Autorino R, De Sio M, Di Lorenzo G, Gallo
L, Damiano R, et al.: Dynamic sentinel node biopsy in clinically node-negative penile cancer versus radical inguinal lymphadenectomy: a comparative study. Urology. 2005; 66: 1282-6.

5. Kroon BK, Horenblas S, Meinhardt W, van der Poel HG, Bex A, van Tinteren H, et al.: Dynamic sentinel node biopsy in penile carcinoma: evaluation of 10 years experience. Eur Urol. 2005; 47: 601-6; discussion 606.

6. Richie JP, Steele GS. Neoplasma of the testis. In: Walsh PC, Retik AB,Vaughan (ed.), Wein AJ. Campbell's Urology. Saunders, Philadelphia. 2002; pp. 2876919.

7. Pettaway CA, Pisters LL, Dinney CP, Jularbal F, Swanson DA, von Eschenbach AC, et al.: Sentinel lymph node dissection for penile carcinoma: the M. D. Anderson Cancer Center experience. J Urol. 1995; 154: 1999-2003.

8. Kroon BK, Horenblas S, Lont AP, Tanis PJ, Gallee MP, Nieweg OE: Patients with penile carcinoma benefit from immediate resection of clinically occult lymph node metastases. J Urol. 2005; 173: 816-9.

9. Ferreira U, Reis LO, Ikari LY, da Silva W Jr, Matheus WE, Denardi F, et al.: Extra-anatomical transobturator bypass graft for femoral artery involvement by metastatic carcinoma of the penis: report of five patients. World J Urol. 2008; 26. [Epub ahead of print]

10. Bevan-Thomas R, Slaton JW, Pettaway CA: Contemporary morbidity from lymphadenectomy for penile squamous cell carcinoma: the M.D. Anderson Cancer Center Experience. J Urol. 2002; 167: 1638-42.

11. d'Ancona CA, de Lucena RG, Querne FA, Martins MH, Denardi F, Netto NR Jr: Long-term followup of penile carcinoma treated with penectomy and bilateral modified inguinal lymphadenectomy. J Urol. 2004; 172: 498-501; discussion 501.

12. Hwang RF, Krishnamurthy S, Hunt KK, Mirza N, Ames FC, Feig B, et al.: Clinicopathologic factors predicting involvement of nonsentinel axillary nodes in women with breast cancer. Ann Surg Oncol. 2003; 10: 248-54.

13. Kroon BK, Horenblas S, Estourgie SH, Lont AP, Valdés Olmos RA, Nieweg OE: How to avoid false-negative dynamic sentinel node procedures in penile carcinoma. J Urol. 2004; 171: 2191-4.

14. Perdonà S, Autorino R, Gallo L, Di Lorenzo G, Cascini GL, Lastoria F, et al.: Role of dynamic sentinel node biopsy in penile cancer: our experience. J Surg Oncol. 2006; 93: 181-5.

15. Hungerhuber E, Schlenker B, Frimberger D, Linke R, Karl A, Stief CG, et al.: Lymphoscintigraphy in 
penile cancer: limited value of sentinel node biopsy in patients with clinically suspicious lymph nodes. World J Urol. 2006; 24: 319-24.

16. Tabatabaei S, Harisinghani M, McDougal WS: Regional lymph node staging using lymphotropic nanoparticle enhanced magnetic resonance imaging with ferumoxtran-10 in patients with penile cancer. J Urol. 2005; 174: 923-7; discussion 927.

17. Leijte JA, Olmos RA, Nieweg OE, Horenblas S: Anatomical Mapping of Lymphatic Drainage in Penile Carcinoma with SPECT-CT: Implications for the Extent of Inguinal Lymph Node Dissection. Eur Urol. 2008; 19. [Epub ahead of print]

18. Tobias-Machado M, Tavares A, Ornellas AA, Molina WR Jr, Juliano RV, Wroclawski ER: Video endoscopic inguinal lymphadenectomy: a new minimally invasive procedure for radical management of inguinal nodes in patients with penile squamous cell carcinoma. J Urol. 2007; 177: 953-7; discussion 958.
19. Sotelo R, Sánchez-Salas R, Carmona O, Garcia A, Mariano M, Neiva G, et al.: Endoscopic lymphadenectomy for penile carcinoma. J Endourol. 2007; 21: 364-7; discussion 367.

20. Leijte JA, Kroon BK, Valdés Olmos RA, Nieweg OE, Horenblas S: Reliability and safety of current dynamic sentinel node biopsy for penile carcinoma. Eur Urol. 2007; 52: 170-7.

21. Hadway P, Smith Y, Corbishley C, Heenan S, Watkin NA: Evaluation of dynamic lymphoscintigraphy and sentinel lymph-node biopsy for detecting occult metastases in patients with penile squamous cell carcinoma. BJU Int. 2007; 100: 561-5.

22. Spiess PE, Izawa JI, Bassett R, Kedar D, Busby JE, Wong F, et al.: Preoperative lymphoscintigraphy and dynamic sentinel node biopsy for staging penile cancer: results with pathological correlation. J Urol. 2007; 177: 2157-61.
Accepted after revision: August 18, 2008

Correspondence address:

Dr. Leonardo Oliveira Reis

R. Votorantim, 51 / 43

Campinas, SP, 13073-090, Brazil

Fax: + 5519 2121-1978

E-mail: reisleo@unicamp.br

\section{EDITORIAL COMMENT}

The best approach of patients T1-2N0M0 penile squamous cell carcinoma with intermediate and high risk of development of inguinal metastasis remains debatable.

A recent study suggests that Solsona and EUA stratification of risk for metastasis have low accuracy to select patients for inguinal dissection (1). To better access the risk of inguinal involvement, we now have a new instrument to guide the decisions. The nomogram of Ficarra et al. based on data of multicentric Italian group classify patients based in the most important prognostic factors as clinical lymph node stage and pathological data as tumor thickness, growth pattern, grade, lymphatic and vascular embolization, corpora 
cavernosa and spongiosa infiltration (2). External validation for other populations is required for better acceptance of this instrument in clinical practice.

Fifteen to $25 \%$ of these patients have micrometastatic disease at time of local treatment. Radical inguinal lymphadenectomy can be curative for low volume disease but the majority of patients will receive a surgical procedure with morbidity higher than $50 \%$ (3). Wait and see policy has been advocate in the past but some recent data have showed that prophylactic dissection offer survival advantage compared to rescue lymphadenectomy after clinical recurrence (4).

Some authors suggest limited dissections. The reliability of this procedure had been questioned because some series reported up to $15 \%$ of late recurrence in other inguinal regions from initial dissection (5).

Anatomic sentinel lymph node as described by Cabanas has been abandoned because the high false negative results.

Dynamic visualization of lymphatic drainage by blue dye in melanoma patients resulted in a renaissance of the sentinel node concept in penile cancer in the mid-1990s. Some recent data of reference centers world wide showed that this technique has reduced morbidity but controversy remains as regards oncological results. This technique requires that specialists in urology, pathology, and nuclear medicine collaborate closely, and high standards are also essential in quality control (6-8).

The Netherlands data reported suggest that with constant improvements and standardization of the technique it proved possible to reduce the incidence of false-negative results with experience (6). In cohort A (1994 until 2001), 21 of 157 explored groins contained tumor-positive sentinel nodes, and five false-negative procedures were encountered, resulting in a false-negative rate of $19.2 \%$. In cohort B (2001 until 2004), 20 of 105 explored groins contained tumor-positive sentinel nodes, and one procedure was false negative. The false-negative rate was $4.8 \%$. The rate of complications dropped from $10.2 \%$ in cohort A to $5.7 \%$ in cohort B (1).

Data from United Kingdom showed that in 255 sentinel lymph node removed from 143 groins; all excised nodes had taken up the radioactive marker, and the blue dye was evident in $87 \%$. Eighteen of $75(24 \%)$ patients and 21 of 143 groins $(15 \%)$ had a positive sentinel. Six of 143 (4\%) groins developed minor complications. Only one false-negative result was reported at a median (range) follow-up of 11 (224) months (7).

On the other hand, in the MD Anderson (8) experience 6 of 32 groins that showed drainage on preoperative lymphoscintigraphy had inguinal node metastasis, as did 1 of 10 that was drainage negative. The sensitivity of preoperative lymphoscintigraphy drainage for cancer detection was $86 \%$. Using dynamic sentinel node biopsy with blue dye plus radiotracer 5 sentinel lymph nodes were positive for cancer, although 2 false-negative results were obtained. Thus, the sensitivity of dynamic sentinel node biopsy per groin for cancer detection was $71 \%$. Authors believe that preoperative lymphoscintigraphy and dynamic sentinel node biopsy as currently performed remain insufficient for detecting occult inguinal disease. They suggest that superficial lymph node dissection remains the gold standard for detecting inguinal microscopic metastasis in these patients (8).

The authors of this interesting manuscript consider that the false negative was $0 \%$ but 2 patients $(11 \%)$ died of late inguinal recurrence in some area considered to have no metastasis. Due to the design of this study, we do not know which will be the outcome if patients when the sentinel was not detected would be received radical dissection.

Conservative management of inguinal regions have some particular problems in Brazilian population. Due to the low socio economic level of these patients, the follow up is not executed as recommended. We believe that the non detected cases at initial evaluation can result in loose the window of cure.

Based in this discussion of the literature and considering that there are no ideal method to locate inguinal micrometastasis I and my colleagues of ABC Medical School proposed a radical dissection applying the principals of minimally invasive surgery. This procedure was designed to achieve reduction in morbidity without jeopardize the oncological control. Preliminary data obtained in 22 dissected groins in 16 patients followed by 36 months showed reduced morbidity and no recurrence compared to open 
conventional surgery (9). Other multicenter Latin America study has also showed the reduced morbidity of endoscopic approach (10).

\section{REFERENCES}

1. Ficarra V, Novara G, Boscolo-Berto R, Artibani W, Kattan MW: How accurate are present risk group assignment tools in penile cancer? World J Urol. 2008; 17. [Epub ahead of print]

2. Ficarra V, Zattoni F, Artibani W, Fandella A, Martignoni G, Novara G, et al.: Penile Cancer Project Members.Nomogram predictive of pathological inguinal lymph node involvement in patients with squamous cell carcinoma of the penis. J Urol. 2006; 175: 1700-4; discussion 1704-5.

3. Nelson BA, Cookson MS, Smith JA Jr, Chang SS: Complications of inguinal and pelvic lymphadenectomy for squamous cell carcinoma of the penis: a contemporary series. J Urol. 2004; 172: 494-7.

4. Kroon BK, Horenblas S, Lont AP, Tanis PJ, Gallee MP, Nieweg OE: Patients with penile carcinoma benefit from immediate resection of clinically occult lymph node metastases. J Urol. 2005; 173: 816-9.

5. Lopes A, Rossi BM, Fonseca FP, Morini S: Unreliability of modified inguinal lymphadenectomy for clinical staging of penile carcinoma.Cancer. 1996; 77: 2099-102.

6. Leijte JA, Kroon BK, Valdés Olmos RA, Nieweg OE, Horenblas S: Reliability and safety of current dynamic

\section{EDITORIAL COMMENT}

The manuscript reports on the value of sentinel lymph node biopsy using lymphoscintigraphy in patients with penile cancer and at least one negative inguinal region. Lymphoscintigraphy with peritumoral intradermal injection of technetium 99m was used to identify the sentinel lymph node. A comparative study using modified inguinal dissection was performed in these regions regardless of the biopsy results. I believe that it is an experimental method with $66 \%$ sensitivity, and $79.3 \%$ specificity when compared with the modified inguinal lymphadenectomy in this manuscript. I sentinel node biopsy for penile carcinoma. Eur Urol. 2007; 52: 170-7.

7. Hadway P, Smith Y, Corbishley C, Heenan S, Watkin NA: Evaluation of dynamic lymphoscintigraphy and sentinel lymph-node biopsy for detecting occult metastases in patients with penile squamous cell carcinoma. BJU Int. 2007; 100: 561-5.

8. Spiess PE, Izawa JI, Bassett R, Kedar D, Busby JE, Wong F, et al.: Preoperative lymphoscintigraphy and dynamic sentinel node biopsy for staging penile cancer: results with pathological correlation. J Urol. 2007; 177: 2157-61.

9. Tobias-Machado M, Tavares A, Ornellas AA, Molina WR Jr, Juliano RV, Wroclawski ER: Video endoscopic inguinal lymphadenectomy: a new minimally invasive procedure for radical management of inguinal nodes in patients with penile squamous cell carcinoma. J Urol. 2007; 177: 953-7; discussion 958.

10. Sotelo R, Sánchez-Salas R, Carmona O, Garcia A, Mariano M, Neiva G, et al.: Endoscopic lymphadenectomy for penile carcinoma. J Endourol. 2007; 21: 364-7; discussion 367.

\section{Dr. Marcos Tobias-Machado \& Dr. Eduardo Simão Starling Section of Urology ABC Medical School São Paulo, SP, Brazil E-mail: tobias-machado@uol.com.br}

agree with the authors' conclusion that the optimal lymphoscintigraphy technique is still in evolution and requires further optimization at high volume centers. The manuscript is well written and the authors are to be congratulated for their interesting work.

\section{Dr. Antonio Augusto Ornellas Section of Urology National Institute of Cancer Rio de Janeiro, Brazil E-mail:ornellasa@hotmail.com}




\section{EDITORIAL COMMENT}

Sentinel lymph node biopsy (SLNB) is a novel diagnostic modality for the assessment of inguinal lymph nodes in penile cancer patients without palpable inguinal adenopathy. It offers the potential of reduced morbidity without compromising cancer detection accuracy as compared to modified inguinal lymph node dissection. However, while it is true some groups such as the Netherlands Cancer Institute (references 7 and 11) have shown promising results with SLNB, its cancer detection accuracy has been shown to be operator dependent and associated with a steep learning curve. This is why the reported false negative rate for cancer detection has ranged from 0 to $22 \%$ (depending on the reported series). Before establishing SLNB as the "gold standard" for the diagnostic evaluation of the inguinal region of penile cancer patients without palpable adenopathy, its results must be more consistently reported across multiple centers and its reduced morbidity versus modified inguinal lymph node dissection must be confirmed. Similarly, emerging diagnostic modalities including video endoscopic inguinal lymph node dissection and nanoparticle MRI may prove superior to SNLB in terms of cancer detection and surgical morbidity. As such, recent advances in radiologic imaging and minimally invasive surgical approaches offer the potential to redefine the diagnostic and treatment standards for the management of penile cancer patients.

\section{Dr. Philippe E. Spiess Department of Interdisciplinary Oncology Moffitt Cancer Center Tampa, Florida, USA E-mail:Philippe.Spiess@moffitt.org}

\section{REPLY BY THE AUTHORS}

In the first editorial comment, the authors state in the 10th paragraph that 2 patients $(11 \%)$ died of late inguinal recurrence in some area considered to have no metastasis. However, according to paragraph 13 in results section of article, both patients presented positive groins at initial diagnosis and were submitted to radical lymphadenectomy. So, these patients were not considered as method fail.

We recommend radical lymphadenectomy when the sentinel lymph node was not detected, due to a possible erratic drainage caused by blockage due to a grossly involved impalpable metastatic lymph node. There are limitations to a less aggressive approach and patients with undetected sentinel lymph node are considered a method limitation, which explains in part $0 \%$ false negative and $66 \%$ sensitivity in the article. The optimal lymphoscintigraphy technique is still in evolution and requires further optimization associated with advances in radiologic imaging and minimally invasive surgical approaches.

Emerging diagnostic modalities including video endoscopic inguinal lymph node dissection, nanoparticle MRI and sentinel lymph node biopsy may prove superior or either complementary among others in terms of cancer detection and surgical morbidity.

The Authors 